\title{
In Silico Drug Screen in Mouse Liver Identifies Candidate Calorie Restriction Mimetics
}

\author{
Kristen Fortney, Eric K. Morgen, ${ }^{2,3}$ Max Kotlyar, ${ }^{1}$ and Igor Jurisica, ${ }^{1,4,5}$
}

\begin{abstract}
Calorie restriction (CR) extends life span in mammals and delays the onset of age-related diseases, including cancer and diabetes. Drugs that target the same genes and pathways as CR may have enormous therapeutic potential. Recently, genome-scale data on the responses of human cell lines to over 1,000 drug treatments have become available. Here we integrate these data with gene expression signatures of $\mathrm{CR}$ in mouse liver to generate a prioritized list of candidate CR mimetics. We identify 14 drugs that reproduce the effects of CR at the transcriptional level.
\end{abstract}

\section{Introduction}

D RECT SCREENS FOR TESTING THE EFFECT OF DRUGS On mouse life span, in which mice are treated with a drug, tracked for several years, and their life span curve compared with that of control animals, are time-consuming, expensive, and methodologically challenging. ${ }^{1}$ As a consequence, few drugs have been screened in this way. For example, only 17 compounds have been (or are being) evaluated as part of the National Institute on Aging's Intervention Testing Program. ${ }^{2}$

Because of these limitations, there is a pressing need for faster and higher-throughput surrogate assays to help identify and prioritize new drug candidates for health span extension. A promising alternative to direct screening of life span is expression-based screening for calorie restriction (CR) mimetics. ${ }^{3-6} \mathrm{CR}$ is one of the most reproducible and effective life span interventions; it extends life span in model organisms from yeast to mammals and delays the age of onset for many diseases of aging, including cancer and diabetes. ${ }^{7}$ Thus, drugs that mimic CR at the transcriptional level may be of great therapeutic value. ${ }^{8}$ In an expression-based screen, mice are treated with drug, tissue samples are taken, and gene expression changes measured using microarrays and compared to those induced by CR. Recent screens found that the drugs metformin and resveratrol reproduce many gene changes seen with $\mathrm{CR}^{4,9,10}$

In this work, we develop an in silico version of expressionbased drug screening for CR mimetics that allows us to test hundreds of drugs at once. We collect nine previously published transcriptional signatures of $\mathrm{CR}$ in mouse liver and screen each one against the Connectivity Map, ${ }^{11}$ a public resource containing genome-scale data on the responses of human cell lines to over 1,000 drug treatments. We then conduct a meta-analysis and identify 14 drugs that consistently rank among the top $\mathrm{CR}$ mimics across multiple studies.

\section{Materials and Methods}

\section{Code}

Code for all analyses was written in $\mathrm{R}$ 2.13.0. Several Bioconductor 2.8 packages were used; we normalized raw Affymetrix CEL files with affy, ${ }^{12}$ used $\operatorname{limm}^{13}$ to identify differentially expressed probesets, and converted mouse IDs [array-specific, GenBank, or Mouse Genome Informatics (MGI)] to human HG-U133A probeset IDs for Connectivity Map analysis using annotationTools. ${ }^{14}$ The drug-drug network was visualized using NAViGaTOR 2.2.1. ${ }^{15}$

\section{Drug-drug interaction network}

We downloaded the DN drug-drug interaction network, where two drugs share an edge if they share a common mode of action, from Mode of Action by NeTwoRk Analysis (MANTRA) ${ }^{16}$

\section{Acquiring transcriptional signatures of $C R$}

We collected gene signatures of CR from published studies. ${ }^{3,4,17-22}$ Our analysis pipeline differed depending on whether the source publications made their raw data available, as described below.

\footnotetext{
${ }^{1}$ Department of Medical Biophysics, ${ }^{2}$ Department of Laboratory Medicine and Pathobiology, and ${ }^{4}$ Department of Computer Science, University of Toronto, Ontario, Canada.

${ }^{3}$ Department of Pathology, University Health Network, Toronto, Ontario, Canada.

${ }^{5}$ Ontario Cancer Institute, University Health Network, and the Campbell Family Institute for Cancer Research, Ontario, Canada.
} 
Identifying differentially expressed genes from raw microarray data. For publications where Affymetrix CEL files were available, ${ }^{4,17,18}$ we reanalyzed the raw data to derive lists of genes significantly up- or downregulated following CR. We normalized CEL files using the robust multiarray averaging (RMA) method $^{23}$ implemented in affy, ${ }^{12}$ and identified differentially expressed probe sets using the empirical Bayes method in limma. ${ }^{13}$

Curating differentially expressed gene lists from published papers. For publications where no raw Affymetrix data were available, ${ }^{3,19-22}$ we downloaded lists of genes reported by the authors to be differentially expressed (in paper text, tables, or supplementary materials). Where fold change and false discovery rate (FDR)-corrected $P$ values were available, we used these data to filter gene lists.

For both types of signature, we removed genes with FDR values greater than 0.05 and (positive or negative) fold change less than 1.25, sorted the remaining genes by FDR, and retained only the top 250 upregulated and the top 250 downregulated genes for further analysis.

\section{Connectivity map analysis of CR signatures}

Mapping mouse CR signatures to human probeset IDs. We mapped mouse gene IDs to human Affymetrix HGU133A IDs for connectivity map analysis following previously established protocols. ${ }^{11}$ First, mouse CR signature genes were mapped to Entrez Gene IDs using either the org.Mm.eg.db Bioconductor 2.8 library (for GenBank or MGI identifiers) or Affymetrix annotation files Mouse430_2.na31 .annot.csv or MG_U74Av2.na32.annot.csv (for probeset IDs). Mouse Entrez Gene IDs were then mapped to human Entrez Gene IDs using homologene.data (release 65; www .ncbi.nlm.nih.gov/homologene), and finally to HGU133A IDs using the Affymetrix annotation file HG-U133A.na31. annot.csv.

Acquiring drug connectivity scores for CR signatures. For each CR signature, mean connectivity scores for 1,309 drugs were calculated as previously described ${ }^{11}$ using data on 6100 drug treatments downloaded from Connectivity Map build 02 at www.broadinstitute.org/cmap/. Drug mean connectivity scores were then converted to ranks. The connectivity score quantifies the extent to which a drug treatment mimics the query signature and is based on the Kolmogorov-Smirnov statistic.

\section{Meta-analysis of drug-response data}

Combining ranked lists of drugs to identify CR mimetics (ranked connectivity scores). We adapted the Rank Product method $^{24}$ to identify drugs that consistently mimic CR at the transcriptional level. For each drug, we calculated the product of its ranks in all CR signatures.

Computing p-values. We randomly permuted the assignment of connectivity scores to drug treatments for the 6100 instances, recalculated mean connectivity scores and drug ranks for 1309 drugs in each signature, and calculated randomized rank products 10,000 times to estimate p-values and false discovery rates.

\section{Results}

\section{Transcriptional signatures of $C R$}

We obtained nine transcriptional signatures of CR in mouse liver by collecting and analyzing data from eight previous publications (we collected two signatures from Tsuchiya et al., ${ }^{18}$ one for wild-type and one for dwarf mice). The mice used to generate the CR signatures came from both sexes and a variety of ages and genetic backgrounds, and CR mice consumed between $56 \%$ and $70 \%$ of the calories of the matched control group, depending on the study. For each mouse CR signature, we constructed an orthologous human signature made up of Affymetrix HG-U133A probe IDs for Connectivity Map analysis (see Materials and Methods). For each human CR signature, we calculated mean connectivity scores for the 1,309 drugs in the Connectivity Map collection. ${ }^{11}$ Connectivity scores ranged between -1 and 1 ; a high, positive mean connectivity score indicates that drug treatment reproduces many of the gene changes with CR. For each human signature, we then constructed a ranked list of drugs based on the connectivity scores.

\section{Meta-analysis identified 14 candidate CR mimetics}

We combined the nine ranked lists of drugs into a single matrix and identified drugs that were consistently highly ranked across signatures using the Rank Product method ${ }^{24}$ (see Materials and Methods). At a false-discovery rate cutoff of $25 \%$ (corresponding to unadjusted $p$ values $<=0.0026$ ), we found that 14 drugs significantly mimic the $C R$ response in mouse liver (Fig. 1A).

Although most signatures were consistent, i.e., gave high ranks to most of the significant drugs (Fig. 1A), two signatures stood out (the two leftmost columns); these corresponded to the earliest signature included in this study ${ }^{20}$ and the signature derived from dwarf mice on a CR diet. ${ }^{18}$ These two relative outliers highlight the usefulness of meta-analyses that identify consistent trends.

Many of the significant drugs show overlapping modes of action (MoA); 10 of 14 form a connected component in the MoA drug-drug interaction network (downloaded from MANTRA $^{16}$ ) where two drugs were joined by an edge if both drug treatments induced significantly similar gene changes (Fig. 1B). We also queried the network with the three best-known longevity therapeutics (metformin, rapamycin, and resveratrol) and found that one of the drugs identified in our screen have MoA similar to resveratrol, and two to rapamycin (Fig. 1B).

Significant drugs (Fig. 1A) are indicated for a wide variety of diseases. For example, pioglitazone is a prescription drug used to treat type 2 diabetes; ${ }^{25}$ colchicine is used to treat gout; ${ }^{26} \mathrm{MG}-262$ is a proteasome inhibitor with anti-inflammatory effects in the heart; ${ }^{27}$ and Gly-His-Lys can activate wound repair. ${ }^{28}$ Three of the drugs have been previously linked to aging: the PI3K inhibitors wortmannin and LY-294002 and the anti-diabetes drug pioglitazone increase lifespan in Drosophila. ${ }^{29,30}$ Other drugs, such as the Chembridge compounds 5155877 and 5224221, are not yet well characterized in terms of their biological effects.

To our knowledge, none of the significant drugs identified in this study has yet been evaluated as a CR mimetic; they should be prioritized for further analyses and biological validation. 
A
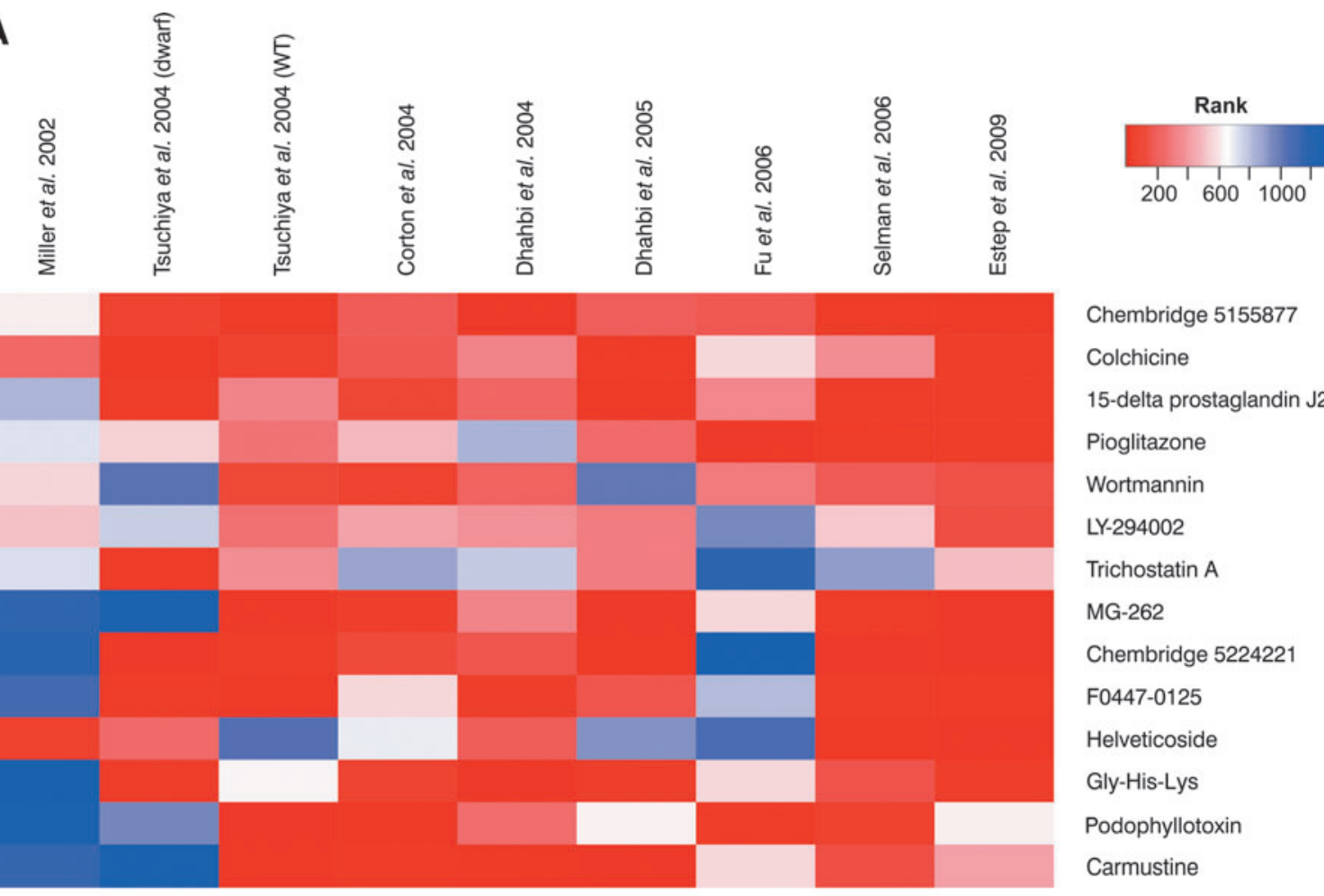

Chembridge 5155877

Colchicine

15-delta prostaglandin J2

Pioglitazone

Wortmannin

LY-294002

Trichostatin A

MG-262

Chembridge 5224221

F0447-0125

Helveticoside

Gly-His-Lys

Podophyllotoxin

Carmustine

B

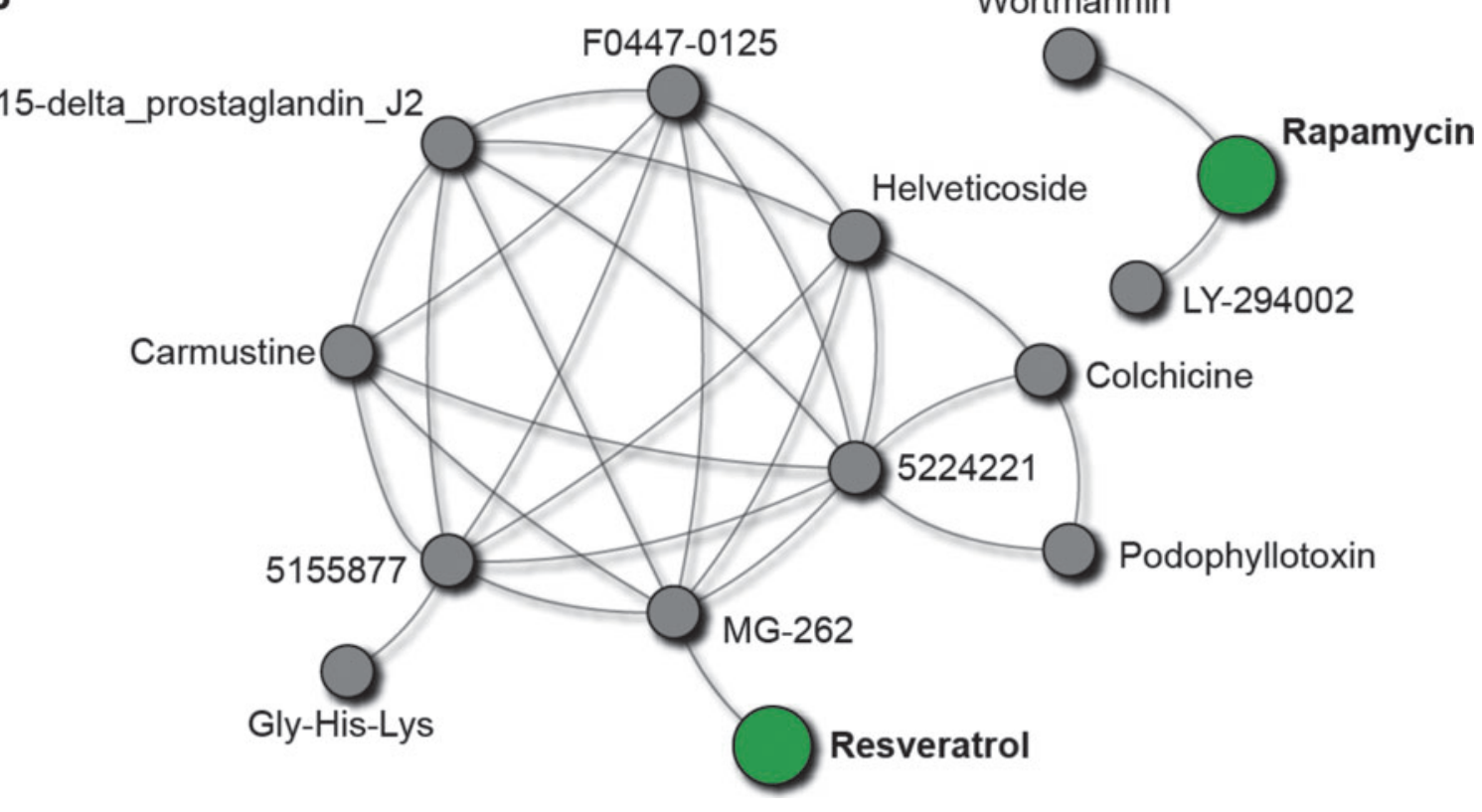

FIG. 1. Fourteen drug treatments significantly mimic the effects of CR on hepatic gene expression. (A) Significant drugs and a heat map showing the rank of each drug in each CR signature queried (top ranks are shown in red). Source publications for signatures are shown on top and ordered from least to most recent; drugs are shown on the right and ordered from most to least significant. (B) Drug-drug interaction network showing links between significant drugs from our screen (grey) and known longevity drugs resveratrol and rapamycin (green). Two drugs are linked if they show similar modes of action. ${ }^{16}$

\section{Discussion}

Whereas few drugs have been directly tested for their effect on mouse life span, over 1,000 drugs have been characterized in terms of their effects on gene expression, and these data are in the public domain. We have applied this resource to identify 14 drugs that have similar transcriptional signatures to CR in mouse liver.

Several dozen other transcriptional signatures of $\mathrm{CR}$ are publicly available, mostly in mouse and rat, but some in primates, including a few in humans. We plan to follow up our pilot study in mouse liver by conducting in silico 
expression-based screening on the full set. Expression-based screening can also be applied to other life-extending treatments for which microarray response data are available, to see, for example, which compounds induce the gene changes seen in long-lived Ames or Snell dwarf mice (versus wild-type).

Longevity drugs have great potential to help treat the diseases of aging, yet few such drugs are known. Ours and similar approaches that leverage the large quantity of public data on drugs and mammalian aging can accelerate the identification and development of new longevity therapeutics.

\section{Author Disclosure Statement}

The authors have no conflict of interest to disclose.

\section{Acknowledgments}

This work was supported in part by Ontario Research Fund (GL2-01-030), Canada Institutes for Health Research (BIO-99745), the Canada Foundation for Innovation (CFI \#12301 and \#203383), the Canada Research Chair Program, and IBM to I.J., and the Ontario Ministry of Health and Long Term Care. The views expressed do not necessarily reflect those of the OMOHLTC.

\section{References}

1. Spindler SR. Review of the literature and suggestions for the design of rodent survival studies for the identification of compounds that increase health and life span. Age (Dordr) 2011; [epub ahead of print]; DOI: 10.1007/s11357-011-9224-6.

2. Nadon NL, Strong R, Miller RA, et al. Design of aging intervention studies: The NIA interventions testing program. Age (Dordr) 2008;30:187-199.

3. Corton JC, Apte U, Anderson SP, Limaye P, Yoon L, Latendresse J, Dunn C, Everitt JI, Voss KA, Swanson C, Kimbrough C, Wong JS, Gill SS, Chandraratna RA, Kwak MK, Kensler TW, Stulnig TM, Steffensen KR, Gustafsson JA, Mehendale HM. Mimetics of caloric restriction include agonists of lipid-activated nuclear receptors. I Biol Chem 2004;279:46204-46212.

4. Dhahbi JM, Mote PL, Fahy GM, Spindler SR. Identification of potential caloric restriction mimetics by microarray profiling. Physiol Genomics 2005;23:343-350.

5. Spindler SR. Use of microarray biomarkers to identify longevity therapeutics. Aging Cell 2006;5:39-50.

6. Spindler SR, Mote PL. Screening candidate longevity therapeutics using gene-expression arrays. Gerontology 2007;53: 306-321.

7. Fontana L, Partridge L, Longo VD. Extending healthy life span-from yeast to humans. Science 2010;328:321-326.

8. Ingram DK, Zhu M, Mamczarz J, Zou S, Lane MA, Roth GS, deCabo R. Calorie restriction mimetics: an emerging research field. Aging Cell 2006;5:97-108.

9. Swindell WR. Comparative analysis of microarray data identifies common responses to caloric restriction among mouse tissues. Mech Ageing Dev 2008;129:138-153.

10. Pearson KJ, Baur JA, Lewis KN, Peshkin L, Price NL, Labinskyy N, Swindell WR, Kamara D, Minor RK, Perez E, Jamieson HA, Zhang Y, Dunn SR., Shrama K, Pleshko N, Woollett LA, Csiszar A, Ikeno Y, Le Couteur D, Elliott PJ, Becker KG, Navas P, Ingram DK, Wolf NS, Ungvari Z, Sinclar DA, de Cabo R. Resveratrol delays age-related de- terioration and mimics transcriptional aspects of dietary restriction without extending life span. Cell Metab 2008;8: 157-168.

11. Lamb J, Crawford ED, Peck D, Modell JW, Blat IC, Wrobel MJ, Lerner J, Brunet JP, Subramanian A, Ross KN, Reich M, Hieronymus H, Wei G, Armstrong SA, Haggarty SJ, Clemons PA, Wei R, Carr SA, Lander ES, Golub TR. The Connectivity Map: Using gene-expression signatures to connect small molecules, genes, and disease. Science 2006;313:1929_ 1935.

12. Gautier L, Cope L, Bolstad BM, Irizarry RA. affy-analysis of Affymetrix GeneChip data at the probe level. Bioinformatics 2004;20:307-315.

13. Smyth GK. Limma: Linear Models for Microarray Data. In: Gentleman R, Carey V, Huber W, Irizarry R, Dudoit S. (eds). Bioinformatics and Computational Biology Solutions Using $R$ and Bioconductor. Heidelberg: Springer; 2005, pp. 397-420.

14. Kuhn A, Luthi-Carter R, Delorenzi M. Cross-species and cross-platform gene expression studies with the Bioconductor-compliant $\mathrm{R}$ package 'annotationTools.' BMC Bioinformatics 2008;9:26.

15. Brown KR, Otasek D, Ali M, McGuffin MJ, Xie W, Devani B, van Toch IL, Jurisica I. NAViGaTOR: Network Analysis, Visualization and Graphing Toronto. Bioinformatics 2009;25: 3327-3329.

16. Iorio F, Bosotti R, Scacheri E, Belcastro V, Mithbaokar P, Ferriero R, Murino L, Tagliaferri R, Brunetti-Pierri N, Isacchi A, di Bernardo D. Discovery of drug mode of action and drug repositioning from transcriptional responses. Proc Natl Acad Sci USA 2010;107:14621-14626.

17. Selman C, Kerrison ND, Cooray A, Piper MD, Lingard SJ, Barton RH, Schuster EF, Blanc E, Gems D, Nicholson JK, Thornton JM, Partridge L, Withers DJ. Coordinated multitissue transcriptional and plasma metabonomic profiles following acute caloric restriction in mice. Physiol Genomics 2006;27:187-200.

18. Tsuchiya T, Dhahbi JM, Cui X, Mote PL, Bartke A, Spindler SR. Additive regulation of hepatic gene expression by dwarfism and caloric restriction. Physiol Genomics 2004;17: 307-315.

19. Fu C, Hickey M, Morrison M, McCarter R, Han ES. Tissue specific and non-specific changes in gene expression by aging and by early stage CR. Mech Ageing Dev 2006;127: 905-916.

20. Miller RA, Chang Y, Galecki AT, Al-Regaiey K, Kopchick JJ, Bartke A. Gene expression patterns in calorically restricted mice: partial overlap with long-lived mutant mice. Mol Endocrinol 2002;16:2657-2666.

21. Dhahbi JM, Kim HJ, Mote PL, Beaver RJ, Spindler SR Temporal linkage between the phenotypic and genomic responses to caloric restriction. Proc Natl Acad Sci USA 2004; 101:5524-5529.

22. Estep PW, 3rd, Warner JB, Bulyk ML. Short-term calorie restriction in male mice feminizes gene expression and alters key regulators of conserved aging regulatory pathways. PLoS One 2009;4 e5242.

23. Irizarry RA, Bolstad BM, Collin F, Cope LM, Hobbs B, Speed TP. Summaries of Affymetrix GeneChip probe level data. Nucleic Acids Res 2003;31:e15.

24. Breitling R, Armengaud P, Amtmann A, Herzyk P. Rank products: A simple, yet powerful, new method to detect differentially regulated genes in replicated microarray experiments. FEBS Lett 2004;573:83-92.

25. Govindan J, Evans M. Pioglitazone in clinical practice: Where are we now? Diabetes Ther 2012;3:1-8. 
26. Terkeltaub RA, Furst DE, Bennett K, Kook KA, Crockett RS, Davis MW. High versus low dosing of oral colchicine for early acute gout flare: Twenty-four-hour outcome of the first multicenter, randomized, double-blind, placebo-controlled, parallel-group, dose-comparison colchicine study. Arthritis Rheum 2010;62:1060-1068.

27. Ludwig A, Fechner M, Wilck N, Meiners S, Grimbo N, Baumann G, Stangl V, Stangl K. Potent anti-inflammatory effects of low-dose proteasome inhibition in the vascular system. J Mol Med (Berl) 2009;87:793-802.

28. Jagtap P, Soriano FG, Virág L, Liaudet L, Mabley J, Szabó É, Haskó G, Marton A, Lorigados CB, Gallyas FJ, Sümegi B, Hoyt DG, Baloglu E, VanDuzer J, Salzman AL, Southan GJ, Szabó C. Novel phenanthridinone inhibitors of poly(adenosine 5 -diphosphate-ribose) synthetase: potent cytoprotective and antishock agents. Crit Care Med 2002;30:1071-1082.
29. Moskalev AA, Shaposhnikov MV. Pharmacological inhibition of phosphoinositide 3 and TOR kinases improves survival of Drosophila melanogaster. Rejuvenation Res 2010;13:246-247.

30. Jafari M, Khodayari B, Felgner J, Bussel, II, Rose MR, Mueller LD. Pioglitazone: An anti-diabetic compound with anti-aging properties. Biogerontology 2007;8:639-651.

Address correspondence to: Igor Jurisica

IBM Life Sciences Discovery Centre Toronto Medical Discovery Tower, 9-305

101 College Street

Toronto, Ontario M5G 1 L7

Canada

E-mail: juris@ai.utoronto.ca 